\title{
Identification of miRNA-mRNA crosstalk in laryngeal squamous cell carcinoma
}

\author{
YONGHUA FEI ${ }^{1}$, PING GUO ${ }^{1}$, FULING WANG ${ }^{2}, \mathrm{HU} \mathrm{LI}^{1}$, \\ YANHUA LEI ${ }^{1}$, WEI LI ${ }^{1}$, XUEHONG XUN ${ }^{1}$ and FENGXIANG LU ${ }^{1}$ \\ ${ }^{1}$ Department of Otolaryngology, Head and Neck Surgery, The First People's Hospital of Jining; ${ }^{2}$ Department of \\ Obstetrics, The First Maternity and Child Health Hospital of Jining, Jining, Shandong 272000, P.R. China
}

Received October 19, 2016; Accepted June 6, 2017

DOI: $10.3892 / \mathrm{mmr} .2017 .7123$

\begin{abstract}
The aim of the present study was to elaborate the underlying pathogenesis of laryngeal squamous cell carcinoma (LSCC). Micro (mi) RNA and messenger (m) RNA expression profiling of patients with LSCC were downloaded from The Cancer Genome Atlas (TCGA) database. Differentially expressed miRNAs (DEMIs) and differentially expressed mRNAs (DEMs) were identified in LSCC compared to normal control tissues. The DEMs targeted by DEMIs were identified and the negative correlation between DEMs and DEMIs was subjected to visualization. The potential functions of DEMs targeted by DEMIs were annotated in Gene Ontology (GO) and Kyoto Encyclopedia of Genes and Genomes (KEGG) database. A total of 663 dysregulated DEMs (449 upregulated and 214 downregulated) and 33 DEMIs (24 upregulated and 8 downregulated) were identified in LSCC compared with normal controls. 502 negative correlations between DEMIs and DEMs were identified and subjected to construct interaction network. In the network, hsa-miR-486, -34c, -206 and -182 had the highest connectivity with DEMs, and respectively regulated 39, 33, 28 and 27 DEMs. DEMs targeted by DEMIs were significantly enriched in signal transduction, actin binding and extracellular region of GO terms and focal adhesion and extracellular matrix-receptor interaction of KEGG pathways. The present study may provide valuable information for understanding the potential oncogenesis mechanism in LSCC and provide the foundation work for diagnosis biomarkers and therapeutic targets for LSCC.
\end{abstract}

Correspondence to: Dr Ping Guo, Department of Otolaryngology Head and Neck Surgery, The First People's Hospital of Jining, 6 Jiankang Road, Rencheng, Jining, Shandong 272000, P.R. China E-mail: guoping@163.com

Key words: microRNAs, regulatory network, laryngeal squamous cell carcinoma, expression profiling, mRNAs

\section{Introduction}

Head and neck cancer (HNC) is the common and heterogeneous malignancy in the world (1). The subtypes of anatomic neoplasm of HNC are classified as alveolar ridge, base of the tongue, buccal mucosa, floor of the mouth, hard palate, hypopharynx, larynx, lip, oral cavity, oral tongue, oropharynx and tonsil. Laryngeal squamous cell carcinoma (LSCC) is the common malignant neoplasm in the head and neck region. It is reported that the incidence rate of LSCC is 3.5-5.1/100,000 persons, and the mortality rate is $2.0-2.2 / 100,000$ persons in 2012 worldwide (2).

With the development of medical technology, the five-year survival rate of patients with LSCC has been improved in recent years. Nevertheless, most of LSCC patients has lost the opportunity of surgical therapy when LSCC is detected at an advanced stage with lymph node metastasis or distant metastasis (3). Smoking, alcohol consumption, coffee and exposure to diesel exhaust fumes increase the incidence rate of LSCC (4).

MicroRNAs (miRNAs), a group of negative regulators of gene expression of the length of 20-25 nucleotides, have also been displayed to be involved in the LSCC pathogenesis in several published articles (5-8). miR-364a-3p promotes cell growth and metastasis in LSCC by targeting PI3K/AKT signaling pathways (5). Decreased miR-744-3p inhibits LSCC metastasis by inactivating AKT/mTOR and the nuclear factor- $\kappa \mathrm{B}$ signaling cascade (6). The expression level of miR-149 is significantly associated with survival duration of LSCC patients and lower expression of miR-149 in patients has the shorter survival time (7). MiR-34a promoter methylation is remarkably increased in the advanced stage LSCC and contributes to the progression, metastasis and poor survival of LSCC (8). However, the pathogenesis of LSCC and associated signaling pathway remains to be explored.

Next generation sequencing (NGS) has proven to be a powerful tool in delineating gene expression alteration throughout cancer progression. The Cancer Genome Atlas (TCGA; https://tcga-data.nci.nih.gov/tcga/) is a publicly funded project and has produced multidimensional data in the DNA, RNA and protein levels for $>30$ human tumors through large-scale genome sequencing (9). Currently, a number of articles are investigating the aberrant transcription 
of LSCC through microarray analysis $(10,11)$. The aberrant miRNA-mRNA crosstalk in LSCC based on RNA-sequencing retrieved from TCGA has not yet been investigated.

Therefore, the aim of the present study was to determine the pathogenesis of LSCC and to investigate the differences between LSCC and non-neoplastic tissue samples at the mRNA and miRNA expression profiling based on TCGA datasets. The study may be able to provide insights into pathogenesis mechanism and pave the way for the development of novel diagnostic markers and therapeutic targets for patients with LSCC.

\section{Materials and methods}

The Cancer Genome Atlas dataset of laryngeal squamous cell carcinoma. MRNA and miRNA expression profiling of LSCC was downloaded from TCGA (https://tcga-data.nci. nih.gov/tcga/) data portal (12). A total of 528 patients with head and neck squamous cell carcinoma (HNSC) was available in TCGA and the corresponding clinical records were downloaded. The inclusion criteria were set as follows: (1) The subtype of anatomic organ was the larynx; (2) patients without the history of other malignancy; (3) patients without neoadjuvant treatment. A total of 105 patients with LSCC were included into the study. Level 3 mRNA and miRNA datasets of LSCC patients were generated from UNC IlluminaHiseq RNASeqV2 and BCGSC IlluminaHiSeq-miRNASeq, respectively.

Screening of differentially expressed mRNAs and miRNAs in LSCC. The mRNA/miRNA expression level was demonstrated as reads per million mRNA/miRNA mapped data. The differentially expressed mRNAs (DEMs) and differentially expressed miRNAs (DEMIs) between LSCC and normal samples were screened by using DESeq2 repackage in $\mathrm{R}$ language $(13,14) . \mathrm{P}<0.001$ and $\log _{2}$ (Fold change) $\mid>2$ were set as cutoffs.

Heat map analysis. In order to assess the similarity of gene expression patterns between LSCC and normal tissues, the DEMs and DEMIs were subjected to two-way hierarchical clustering analysis. The heat map was drawn by the 'pheatmap' package in R language (15). A dot represented the expression level of a dysregulated DEM/DEMIs in a sample of LSCC/normal tissues.

Prediction of targeted DEMs of DEMIs. Target mRNAs were predicted for DEMs by using miRWalk2 (http://zmf.umm.uni-heidelberg.de/apps/zmf/mirwalk2/)

database (16), in which the interaction between miRNAs and mRNAs is validated by experimental methods, such as western blotting, luciferase reporter gene assays and reverse transcription-quantitative polymerase chain reaction. miRNAs frequently negatively regulate the expression of the targeted mRNA. In the current work, negatively regulatory miRNA-mRNA pairs were screened. Putative targets of DEMIs were predicted by six bioinformatics algorithms covering RNA22 version 2.0 (https://cm.jefferson.edu/rna22v2.0/), miRanda-mirSVR (http://www.microrna.org/), miRDB (http://mirdb.org/miRDB/), miRWalk (http://www.umm. uni-heidelberg.de/apps/zmf/mirwalk/index.html), PICTAR2 (http://pictar.mdc-berlin.de/) and TargetScan version 6.2 (http://www.targetscan.org/). The predictive genes, synchronously retrieved by $>4$ algorithms in miRWalk2.0 database, were selected to compare with the identified DEMs in LSCC and the overlapped genes were considered as the target genes of DEMs.

Construction of miRNA-mRNA regulatory network. Identified DEMI-DEM regulatory pairs were visualized by Cytoscape software (http://cytoscape.org) (17). In the regulatory network, a circular node represented the mRNA and a rectangle node represents the miRNA. The line indicated the association between DEMI and DEM. Red color represented upregulation and green color represented down-regulation.

Functional annotation of DEMs in LSCC. Gene Ontology (GO) terms and the Kyoto Encyclopedia of Genes and Genomes (KEGG) pathway were commonly used to predict the potential functions of DEMs. The functional annotation including biological process, molecular function, cellular component and pathway of DEMs was mapped by using GeneCoDis3 (http://genecodis.cnb.csic.es/analysis) (18). FDR $<0.05$ was set as the cutoff of GO terms and KEGG pathway (19).

\section{Results}

Differentially expressed mRNAs in LSCC. In the present study, level 3 mRNA expression data were downloaded from TCGA data portal (Table I). Differentially expressed analysis was performed between LSCC and normal control samples. Finally, 663 mRNAs were identified as significantly differentially expressed under the cutoff of $\mathrm{P}<0.001$ and $\mid \log 2 \mathrm{FCl}>2$, with 449 up-regulated and 214 downregulated mRNAs. As Table II presents, LAMA1 was the most significantly upregulated DEM in LSCC, with 116-fold upregulation; and KRT4 was the most significantly downregulated DEM in LSCC, with $\sim 85$-fold downregulation.

Differentially expressed miRNAs in LSCC. Level 3 miRNA expression data were downloaded as well as from TCGA data portal (Table I). Differentially expressed miRNAs (DEMIs) were identified between LSCC and normal control samples. A total of 33 DEMIs were identified as the threshold of $\mathrm{P}<0.001$ and $\mid \log 2 \mathrm{FCl}>2$, consisting of 25 up-regulated DEMIs and 8 down-regulated DEMIs. Hierarchical clustering analysis displayed that the expression pattern of 33 DEMIs were discrimination between LSCC and control tissues (Fig. 1). As Table III indicates, hsa-miR-105-1 and hsa-miR-105-2 most significantly upregulated DEMIs in LSCC and hsa-miR-1-2 was significantly downregulated DEMI in LSCC.

Construction of the miRNA-mRNA network. Target genes of 33 DEMIs, predicted using the miRWalk2 database, were overlapped with the DEMs in LSCC. Target genes with significantly differential expression were deemed to the target DEMs of DEMIs. The identified reverse association between DEMs and DEMIs was visualized by Cytoscape software. A total of 502 DEMs-DEMIs pairs were subjected 
Table I. mRNA and miRNA expression profiling datasets of laryngeal squamous cell carcinoma.

\begin{tabular}{llrr}
\hline Data type & Platform & Case & Control \\
\hline mRNA & UNC IlluminaHiseq_RNASeqV2 & 105 & 9 \\
miRNA & BCGSC IlluminaHiSeq-miRNASeq & 95 & 9 \\
\hline
\end{tabular}

mRNA, messenger RNA; miRNA, microRNA.

Table II. Top 15 up- and downregulated differentially expressed mRNAs in laryngeal squamous cell carcinoma.

\begin{tabular}{|c|c|c|}
\hline Gene symbol & Gene ID & $\log _{2} \mathrm{FC}$ \\
\hline \multicolumn{3}{|l|}{ Upregulation } \\
\hline LAMA1 & 284217 & 6.561864 \\
\hline COL11A1 & 1301 & 6.43351 \\
\hline NOTUM & 147111 & 6.143955 \\
\hline FABP4 & 2167 & 6.108568 \\
\hline MMP11 & 4320 & 5.884637 \\
\hline HOXD11 & 3237 & 5.767214 \\
\hline SPRR2G & 6706 & 5.722904 \\
\hline COL22A1 & 169044 & 5.412467 \\
\hline KRT75 & 9119 & 5.392634 \\
\hline FBN2 & 2201 & 5.389671 \\
\hline CXCL5 & 6374 & 5.385775 \\
\hline MMP3 & 4314 & 5.379472 \\
\hline KLHDC7B & 113730 & 5.373686 \\
\hline ESM1 & 11082 & 5.346206 \\
\hline PNCK & 139728 & 5.1961812 \\
\hline \multicolumn{3}{|c|}{ Downregulation } \\
\hline KRT4 & 3851 & -6.4084 \\
\hline MAL & 4118 & -6.01297 \\
\hline ATP2A1 & 487 & -5.80844 \\
\hline PRH1 & 5554 & -5.8001 \\
\hline ENO3 & 2027 & -5.63503 \\
\hline CKM & 1158 & -5.35734 \\
\hline MB & 4151 & -5.26978 \\
\hline MYOM1 & 8736 & -5.22194 \\
\hline KRT78 & 196374 & -5.04242 \\
\hline MYOZ3 & 91977 & -4.99803 \\
\hline FNDC5 & 252995 & -4.98228 \\
\hline PRR4 & 11272 & -4.64908 \\
\hline CMYA5 & 202333 & -4.62633 \\
\hline KRT13 & 3860 & -4.58126 \\
\hline HSPB6 & 126393 & -4.57794 \\
\hline
\end{tabular}

FC, fold change; mRNA, messenger RNA.

to construct the regulatory network. As Fig. 2 reveals, the networks were composed of 245 nodes covering 218 DEMs. In Fig. 2A, the up-regulated DEMIs/downregulated DEMs interaction network included in 142 nodes and 347 edges. hsa-miR-34c and hsa-miR-182 had the highest connectivity
Table III. Dysregulated differentially expressed miRNAs in laryngeal squamous cell carcinoma.

\begin{tabular}{|c|c|c|}
\hline miRNA & $\log _{2} \mathrm{FC}$ & Up/downregulation \\
\hline hsa-miR-105-1 & 7.062514 & Up \\
\hline hsa-miR-105-2 & 6.848488 & Up \\
\hline hsa-miR-1269 & 3.903254 & Up \\
\hline hsa-miR-182 & 2.301083 & Up \\
\hline hsa-miR-183 & 2.310896 & Up \\
\hline hsa-miR-187 & 3.465487 & Up \\
\hline hsa-miR-196a-1 & 4.718663 & $\mathrm{Up}$ \\
\hline hsa-miR-196b & 3.454173 & Up \\
\hline hsa-miR-210 & 2.633137 & Up \\
\hline hsa-miR-31 & 3.14187 & Up \\
\hline hsa-miR-34c & 2.270289 & Up \\
\hline hsa-miR-3607 & 2.663814 & $\mathrm{Up}$ \\
\hline hsa-miR-455 & 2.362365 & Up \\
\hline hsa-miR-508 & 4.415202 & Up \\
\hline hsa-miR-509-1 & 3.7981 & Up \\
\hline hsa-miR-509-2 & 4.157125 & Up \\
\hline hsa-miR-509-3 & 4.030178 & Up \\
\hline hsa-miR-514-1 & 4.747188 & Up \\
\hline hsa-miR-514-2 & 4.80023 & Up \\
\hline hsa-miR-514-3 & 4.646079 & Up \\
\hline hsa-miR-708 & 2.170909 & Up \\
\hline hsa-miR-767 & 6.934857 & Up \\
\hline hsa-miR-9-1 & 4.448849 & Up \\
\hline hsa-miR-9-2 & 4.483711 & $\mathrm{Up}$ \\
\hline hsa-miR-96 & 2.597547 & Up \\
\hline hsa-miR-1-2 & -4.28151 & Down \\
\hline hsa-miR-133a-1 & -3.48784 & Down \\
\hline hsa-miR-133b & -3.82296 & Down \\
\hline hsa-miR-139 & -2.29545 & Down \\
\hline hsa-miR-206 & -2.59706 & Down \\
\hline hsa-miR-375 & -2.38564 & Down \\
\hline hsa-miR-378c & -2.53979 & Down \\
\hline hsa-miR-486 & -2.08201 & Down \\
\hline
\end{tabular}

FC, fold change; miRNA, microRNA.

for DEMs and negatively interacted with 33 and 27 DEMs, respectively. In Fig. 2B, the down-regulated DEMIs/upregulated DEMs interaction network included in 103 nodes and 155 edges, hsa-miR-486 and hsa-miR-206 had the highest 


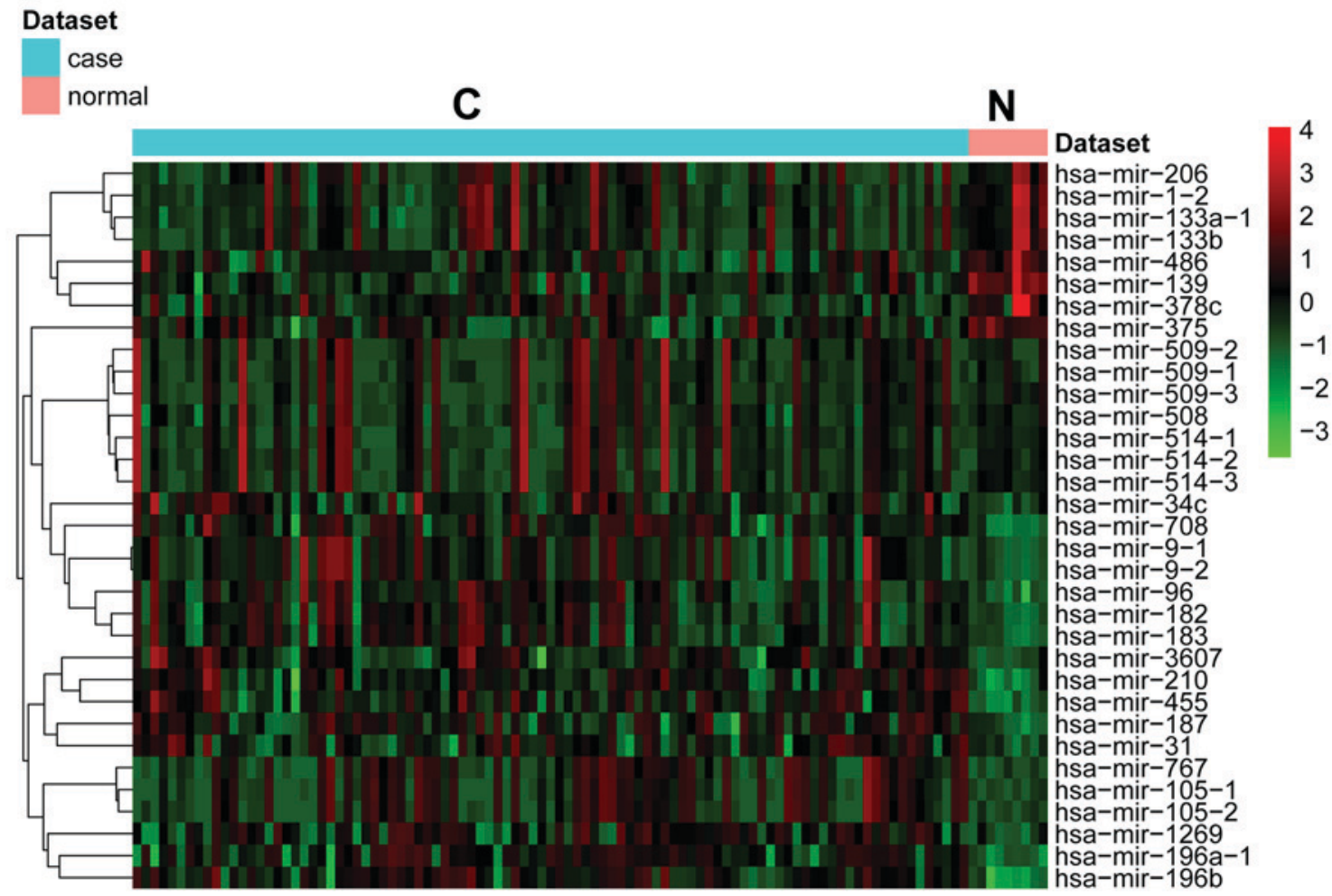

Figure 1. Hierarchical clustering analysis of the expression level of differentially expressed miRNAs between LSCC and normal control tissues. Row and column represented DEMIs and tissue samples. Red and green indicated up- and downregulated DEMIs in LSCC. C represented LSCC and N represented normal control tissues. LSCC, laryngeal squamous cell carcinoma; miR, microRNA.

A

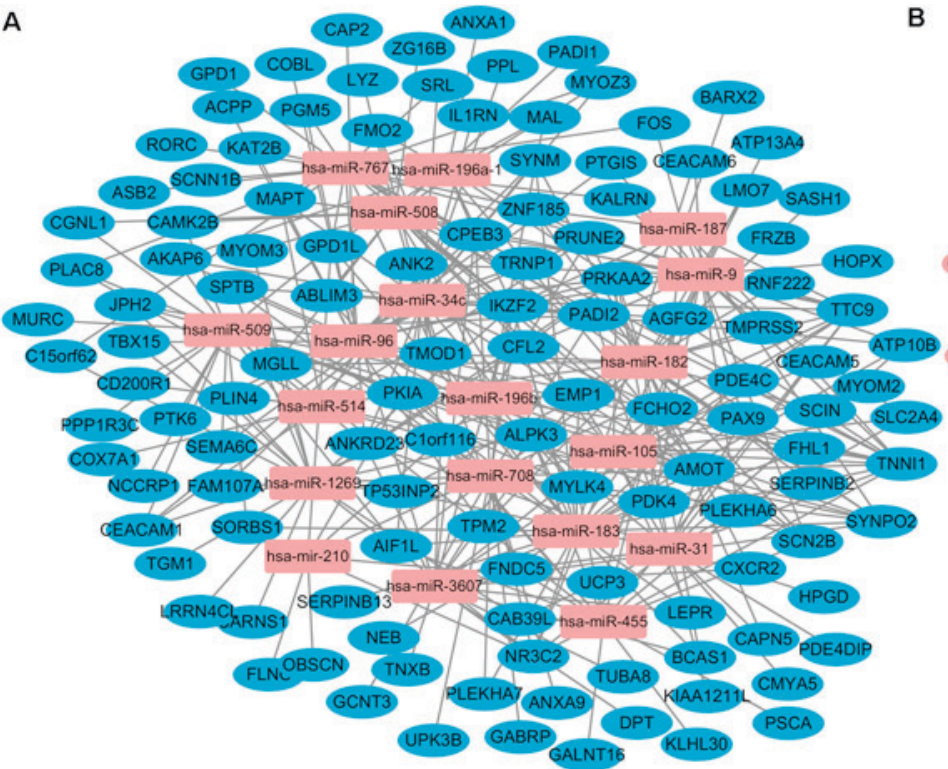

B

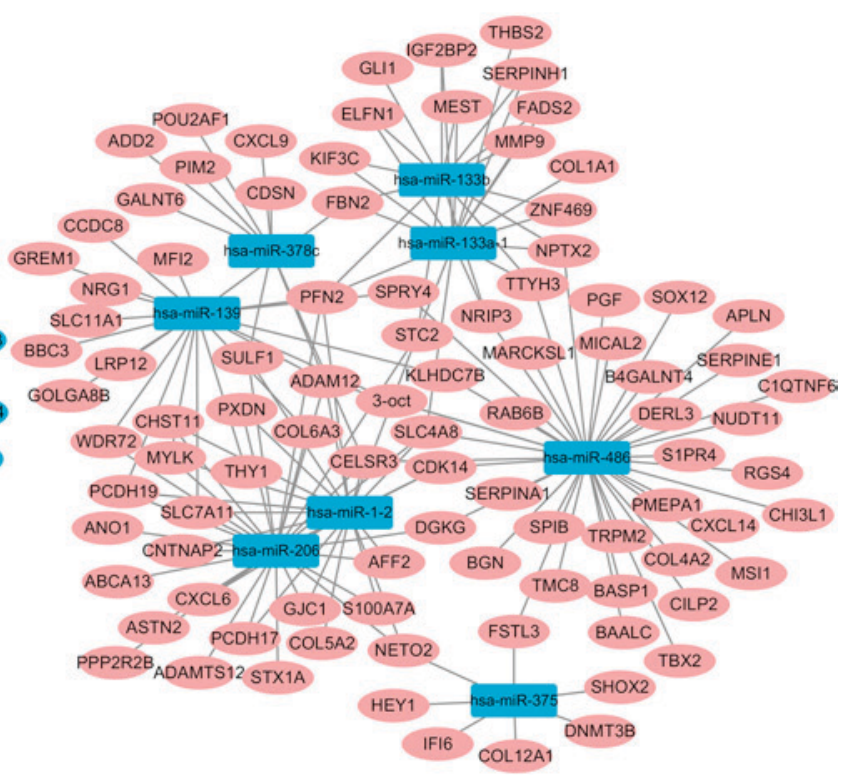

Figure 2. DEMIs-DEMs regulatory network in LSCCs. Circular nodes represented DEMs and rectangle nodes represented DEMIs. Blue color represented downregulation and pink color represented upregulation in LSCC. Solid lines indicated regulatory associations between DEMs and DEMIs. (A) The upregulated DEMIs/downregulated DEMs interaction network. (B) The downregulated DEMIs/up-regulated DEMs interaction network. DEMI, differentially expressed miRNAs; DEMs, differentially expressed mRNAs; LSCCs, laryngeal squamous cell carcinoma.

connectivity for DEMs and negatively interacted with 39 and 28 DEMs, respectively.

GO terms annotation of DEMs targeted by DEMIs in LSCC. In order to predict the 218 DEMs targeted by DEMIs in LSCC, the GO term was annotated. The threshold of biological process, molecular function and cellular component terms of GO was set as FDR<0.05. As Table IV presents, multicellular organismal development $\left(\mathrm{FDR}=6.26 \times 10^{-5}\right)$, blood coagulation $\left(\mathrm{FDR}=6.84 \times 10^{-4}\right)$ and regulation of proteolysis $\left(\mathrm{FDR}=8.09 \times 10^{-4}\right)$ were the most significant enrichment of biological process; actin binding $\left(\mathrm{FDR}=4.30 \times 10^{-9}\right)$, protein 
Table IV. The GO enrichment of differentially expressed mRNAs targeted by differentially expressed miRNAs.

\begin{tabular}{|c|c|c|}
\hline GO ID & GO terms & FDR \\
\hline \multicolumn{3}{|c|}{ Biological process } \\
\hline GO:0007275 & Multicellular organismal development & 0.0000626 \\
\hline GO:0007596 & Blood coagulation & 0.000683755 \\
\hline GO:0030162 & Regulation of proteolysis & 0.000809008 \\
\hline GO:0030199 & Collagen fibril organization & 0.000809008 \\
\hline GO:0030049 & Muscle filament sliding & 0.000869945 \\
\hline GO:0007165 & Signal transduction & 0.000911484 \\
\hline GO:0010951 & Negative regulation of endopeptidase activity & 0.000957166 \\
\hline GO:0014070 & Response to organic cyclic compound & 0.000965062 \\
\hline GO:0030308 & Negative regulation of cell growth & 0.000965062 \\
\hline GO:0006633 & Fatty acid biosynthetic process & 0.000982443 \\
\hline \multicolumn{3}{|c|}{ Molecular function } \\
\hline GO:0003779 & Actin binding & 0.00000000430 \\
\hline GO:0005515 & Protein binding & 0.00000126 \\
\hline GO:0051287 & NAD binding & 0.0000988 \\
\hline GO:0008307 & Structural constituent of muscle & 0.000120396 \\
\hline GO:0005201 & Extracellular matrix structural constituent & 0.00012247 \\
\hline GO:0004674 & Protein serine/threonine kinase activity & 0.000338952 \\
\hline GO:0004867 & Serine-type endopeptidase inhibitor activity & 0.000352194 \\
\hline GO:0005515 & Protein binding & 0.000587697 \\
\hline GO:0003677 & DNA binding & 0.000607957 \\
\hline GO:0005509 & Calcium ion binding & 0.000678006 \\
\hline \multicolumn{3}{|c|}{ Cellular component } \\
\hline GO:0005576 & Extracellular region & 0000000000141 \\
\hline GO:0005737 & Cytoplasm & 0.0000000139 \\
\hline GO:0005886 & Plasma membrane & 0.0000000150 \\
\hline GO:0031012 & Extracellular matrix & 0.0000000356 \\
\hline GO:0030018 & $\mathrm{Z}$ disc & 0.000000106 \\
\hline GO:0005737 & Cytoplasm & 0.000000273 \\
\hline GO:0005576 & Extracellular region & 0.000000343 \\
\hline GO:0005615 & Extracellular space & 0.000000525 \\
\hline GO:0005856 & Cytoskeleton & 0.00000168 \\
\hline GO:0005578 & Proteinaceous extracellular matrix & 0.00000720 \\
\hline
\end{tabular}

FDR, false discovery rate; GO, Gene Ontology; mRNA, messenger RNA; miRNA, microRNA.

binding $\left(\mathrm{FDR}=1.26 \times 10^{-6}\right)$ and NAD binding $\left(\mathrm{FDR}=9.88 \times 10^{-5}\right)$ were the highest enrichment of molecular function; extracellular region $\left(\mathrm{FDR}=1.41 \times 10^{-10}\right)$, cytoplasm $\left(\mathrm{FDR}=1.39 \times 10^{-8}\right)$ and plasma membrane $\left(\mathrm{FDR}=1.50 \times 10^{-8}\right)$ were the highest enrichment of cellular component.

KEGG signaling pathway enrichment. KEGG enrichment analysis was performed to understand the signaling pathways of DEMs involved in LSCC. A total of 214 out of 218 DEMs targeted by DEMIs in LSCC were significantly enriched in 13 signaling pathways, including focal adhesion (hsa04510), extracellular matrix (ECM)-receptor interaction (hsa04512), mTOR signaling pathway (hsa04150) and cytokine-cytokine receptor interaction (hsa04060), as Table V indicates.

\section{Discussion}

In order to improve understanding of LSCC, the mRNA and miRNA expression profiling of LSCC, derived from TCGA database, was subjected to integrate analysis and miRNA-mRNA crosstalk analysis in the current study.

Zhang et al (20) obtains the miRNA and mRNA expression profiling through high-throughput from 10 LSCC samples and 2 healthy samples and construct the miRNA-mRNA crosstalk in LSCC. In the published paper (20), miR-182, miR-183 and miR-96 are identified as significantly upregulated in LSCC, which is consistent with the present analysis. Whereas, miR-1301, miR-184 and miR-224 are dysregulated in LSCC based on the analysis by Zhang et al (20), but those miRNAs are not dysregulated in LSCC in our work. The discrimination 
Table V. KEGG pathway enrichment of differentially expressed mRNAs targeted by in laryngeal squamous cell carcinoma.

\begin{tabular}{llll}
\hline KEGG ID & \multicolumn{1}{c}{ KEGG term } & \multicolumn{1}{c}{ FDR } & \multicolumn{1}{c}{ Genes } \\
\hline hsa04510 & Focal adhesion & 0.0000291 & PGF, MYLK, COL4A2, FLNC, TNXB, COL5A2, \\
& & & COL1A1, THBS2, COL6A3 \\
hsa04512 & ECM-receptor interaction & 0.0000322 & COL4A2, TNXB, COL5A2, COL1A1, THBS2, COL6A3 \\
hsa04974 & Protein digestion and absorption & 0.0000632 & COL4A2, COL5A2, COL1A1, COL6A3 \\
hsa05146 & Amoebiasis & 0.000313134 & COL4A2, COL5A2, COL1A1 \\
hsa04974 & Protein digestion and absorption & 0.000506167 & COL4A2, COL12A1, COL5A2, COL1A1, COL6A3 \\
hsa04062 & Chemokine signaling pathway & 0.00169738 & CXCL6, CXCL9, CXCR2, CXCL14 \\
hsa04150 & mTOR signaling pathway & 0.0101123 & PGF, CAB39L, PRKAA2 \\
hsa04920 & Adipocytokine signaling pathway & 0.0181962 & LEPR, SLC2A4, PRKAA2 \\
hsa04910 & Insulin signaling pathway & 0.0188236 & SLC2A4, PRKAA2, SORBS1, PPP1R3C \\
hsa00564 & Glycerophospholipid metabolism & 0.0232727 & GPD1, GPD1L, DGKG \\
hsa05323 & Rheumatoid arthritis & 0.0251295 & CXCL6, FOS, PGF \\
hsa04666 & Fc $\lambda$ R-mediated phagocytosis & 0.0294864 & CFL2, SCIN, MARCKSL1 \\
hsa04060 & Cytokine-cytokine receptor interaction & 0.0324164 & CXCL6, CXCL9, LEPR, CXCR2, CXCL14
\end{tabular}

FDR, false discovery rate; KEGG, Kyoto Encyclopedia of Genes and Genomes; ECM, extracellular matrix.

between the results of Zhang et al (20) and the present study may be attributed to the different LSCC samples for research. On one hand, 12 experssion profiles were subjected to bioinformatics analysis in the study by Zhang et al (20), whereas in the present study, 114 profiles were analyzed. On the other hand, LSCC samples in the study by Zhang et al (20) were obtained from China, whereas the samples in the present paper were derived from America and Asia, according to TCGA database.

hsa-miR-486 reported the highest connectivity with target genes and negatively regulated 39 DEMs expression in the regulatory network, such as COL4A2, DGKG, PGF, MARCKSL1, CXCL14 and KLHDC7B. These protein-coding genes were significantly up-regulated in LSCC and enriched in 12 signaling pathways (Table V), covering focal adhesion, ECM-receptor interaction, mTOR signaling pathway and cytokine-cytokine receptor interaction. miR-486 functions as a tumor suppressor in several types of tumor covering colorectal cancer (CRC), papillary thyroid carcinoma (PCT) and lung cancer (21-23). miR-486-5p is downregulated in laryngeal carcinoma by microarray (24) and the roles of miR-486-5p in LSCC are unclear. miR-486-5p is downregulated in CRC tissues compared with the adjacent non-tumor tissues and the CRC mice mode presents increased miR-486-5p suppresses tumor growth and lymphangiogenesis (21). miR-486-5p is significantly downregulated in PCT tissues and cell lines and its underexpression promotes cell proliferation and represses cell apoptosis in PTC (22). It is reported that miR-486-5p is downregulated in lung adenocarcinoma and COL4A2 is upregulated in non-small cell lung cancer and small cell lung cancer $(23,25)$. COL $4 A 2$, encodes for the collagen type IV alpha 2 chain, and is the subunit of type IV collagen, which is the major structural component of basement membranes. Abnormally expressed type IV collagen is correlated to tumor spreading and migration (26). Upregulation of COL4A2 is involved in anoikis resistance in epithelia ovarian cancer (27). $P G F$, encodes the placenta growth factor, which belongs to vascular endothelial growth factor (VEGF) family of angiogenic factors. The VEGF family serves key roles in tumor angiogenesis. It is reported that PGF is over expressed in head and neck squamous cell carcinoma, which is involved in angiogenesis (28). PGF is upregulated in breast cancer and the increased expression of PGF is associated with recurrence, metastasis and poor prognosis in patients with breast cancer (29). In prostate cancer, the expression of CXCL14 is positively correlated with tumor aggressiveness (30).

hsa-miR-34c was significantly upregulated and negatively regulated 33 DEMs in LSCC, such as PRKAA2, MYOZ3 and FNDC5. It is reported that hsa-miR-34c is significantly downregulated in various tumors and acts as a tumor suppressor in NSCLC, endometrial carcinoma (EC), nasopharyngeal carcinoma (NPC) and osteosarcoma (OS) (31-34). Increased expression of miR-34c-3p inhibits cell proliferation, migration and invasion in NSCLC by targeting eIF4E (31). Upregulated miR-34c inhibits cell proliferation and colony formation in EC by targeting E2F3 (32). The promoter region of miR-34c is hypermethylated and miR-34c suppresses tumor growth and metastasis in NPC by targeting MET (33). miR-34c impedes OS metastasis and chemo-resistance by targeting Notch 1 and LEF1 (34). As the target genes of miR-34c, MYOZ3 and FNDC5 were in the top 15 downregulated DEMs in LSCC (Table II). FNDC5 encodes a secreted protein, named fibronectin type III domain containing 5. Serum level of FNDC5 is significantly lower in patients with breast cancer compared with healthy volunteers and it is the independent risk factor of breast cancer (35). MYOZ3 encodes myozenin 3, which belongs to myozenin family. However, the biological functions of MYOZ3 in tumor process remain unclear. To the best of the authors' knowledge, the present study is the first to report that MYOZ3 is involved in LSCC oncogenesis. PRKAA2 encodes protein kinase AMP-activated catalytic subunit alpha 2, which belongs to the Ser/Thr protein kinase family and was significantly enriched in mTOR signaling pathway and adipocytokine 
signaling pathway (Table V). Except for miR-34c, PRKAA2 was regulated by miR-105, miR-182 and miR-96.

hsa-miR-1-2 and hsa-miR-105-1 were the most significantly down- and upregulated DEMI in LSCC compared with normal controls, respectively. miR-1 functions as a tumor suppressor in various cancers. miR-1 and miR-133a inhibit cell proliferation, invasion and increases cell apoptosis in bladder cancer cells by downregulation of PTMA and PNP (36). Decreased miR-1/133a cluster promotes cell migration and invasion in lung squamous cell carcinoma by targeting coronin 1C (37). Moreover, miR-1 is downregulated and regulates focal adhesion and ECM-receptor interaction pathways in head and neck squamous cell carcinoma (38). miR-105 is characteristically expressed in metastatic breast cancer cells compared with non-metastatic cancer cells and over expression of miR-105 in non-metastatic cancer induces metastasis and vascular permeability (39). Upregulation of miR-105 is correlated with gastric cancer (40).

MMP9 encodes matrix metalloproteinase 9, which belongs to the MMP family and is involved in the breakdown of extracellular matrix in disease processes, such as tumor metastasis. MMP1, MMP3 and MMP11 were also significantly upregulated in LSCC. The expression level of MMP9 is positively correlated with lymph-node metastasis and TNM stage in LSCC (41). In the miRNA-mRNA crosstalk, MMP9 was negatively regulated by miR-133a-1 and miR-133b. Saito et al (42) indicates that miR-133b had the lower expression in LSCC compared with controls, which is in accordance with the current analysis. miR-133a-1 was another negative regulator of MMP9. miR-1-2 and miR-133a-1 are located on 18q11.2 cluster; miR-1-1 and miR-133a-2 are located on 20q13.33 cluster. In bladder cancer, miR-1 and miR-133a are involved in tumor processes including proliferation, invasion and apoptosis (36). The roles of miR-133a in LSCC are as yet unreported.

Focal adhesion (hsa04510) and ECM-receptor interaction (hsa04512) was the most significantly dysregulated signaling pathway in LSCC. Focal adhesion serves key roles in cell motility, proliferation and cell survival of various cancers, such as triple-negative breast cancer, endometrial cancer and pancreatic ductal adenocarcinoma (43-45). The ECM contributes to cell morphogenesis and function. ECM participates in cell adhesion, migration, differentiation, proliferation and apoptosis through interaction with some of transmembrane molecules. ECM-receptor interactions are dysregulated in colorectal cancer, clear renal cell carcinoma and esophageal squamous cell carcinoma (46-48). It is reported that focal adhesion and ECM-receptor interaction pathways are dysregulated in head and neck squamous cell carcinoma (38). Based on the aforementioned, focal adhesion and ECM-receptor interactions may be involved in the tumor biology including cell growth, invasion, motility and metastasis of LSCC.

In conclusion, availably public mRNA and miRNA expression profiling of LSSC, derived from TCGA database, was subjected to bioinformatics analysis. The miRNA-mRNA crosstalk was constructed and a set of key dysregulated miRNAs and signaling pathways were identified in LSCC. The hope is that the current study may be helpful for understanding the underlying oncogenesis mechanism in LSCC and provide the foundation work for diagnosis biomarkers and therapeutic targets for LSCC.

\section{References}

1. Parkin DM, Bray F, Ferlay J and Pisani P: Global cancer statistics, 2002. CA Cancer J Clin 55: 74-108, 2005.

2. Siegel R, Naishadham D and Jemal A: Cancer statistics, 2012. CA Cancer J Clin 62: 10-29, 2012.

3. Simard EP, Torre LA and Jemal A: International trends in head and neck cancer incidence rates: Differences by country, sex and anatomic site. Oral Oncol 50: 387-403, 2014.

4. Vassileiou A, Vlastarakos PV, Kandiloros D, Delicha E, Ferekidis E, Tzagaroulakis A and Nikolopoulos TP: Laryngeal cancer: Smoking is not the only risk factor. B-Ent 8: 273-278, 2012.

5. Geng J, Liu Y, Jin Y, Tai J, Zhang J, Xiao X, Chu P, Yu Y, Wang SC, Lu J, et al: MicroRNA-365a-3p promotes tumor growth and metastasis in laryngeal squamous cell carcinoma. Oncol Rep 35: 2017-2026, 2016.

6. Li JZ, Gao W, Lei WB, Zhao J, Chan JY, Wei WI, Ho WK and Wong TS: MicroRNA 744-3p promotes MMP-9-mediated metastasis by simultaneously suppressing PDCD4 and PTEN in laryngeal squamous cell carcinoma. Oncotarget 7: 58218-58233, 2016.

7. Xu Y, Lin YP, Yang D, Zhang G and Zhou HF: Clinical significance of mir-149 in the survival of patients with laryngeal squamous cell carcinoma. Biomed Res Int 2016: 8561251, 2016.

8. Shen Z, Zhou C, Li J, Ye D, Li Q, Wang J, Cui X, Chen X, Bao T and Duan S: Promoter hypermethylation of miR-34a contributes to the risk, progression, metastasis and poor survival of laryngeal squamous cell carcinoma. Gene 593: 272-276, 2016.

9. Cancer Genome Atlas Research Network, Weinstein JN, Collisson EA, Mills GB, Shaw KR, Ozenberger BA, Ellrott K, Shmulevich I, Sander C and Stuart JM: The cancer genome atlas pan-cancer analysis project. Nat Genet 45: 1113-1120, 2013.

10. Yang B and Bao X: Identification of genes associated with laryngeal squamous cell carcinoma samples based on bioinformatic analysis. Mol Med Rep 12: 3386-3392, 2015.

11. Ma LJ, Li W, Zhang X, Huang DH, Zhang H, Xiao JY and Tian YQ: Differential gene expression profiling of laryngeal squamous cell carcinoma by laser capture microdissection and complementary DNA microarrays. Arch Med Res 40: 114-123, 2009.

12. Tomczak K, Czerwińska P and Wiznerowicz M: The Cancer Genome Atlas (TCGA): An immeasurable source of knowledge. Contem Oncol (Pozn) 19: A68-A77, 2015.

13. Gentleman R, Carey V, Huber W, Irizarry R and Dudoit S (eds): Bioinformatics and computational biology solutions using $\mathrm{R}$ and Bioconductor. Springer, New York, 2005.

14. Love MI, Huber W and Anders S: Moderated estimation of fold change and dispersion for RNA-seq data with DESeq2. Genome Biol 15: 550, 2014

15. Zhao S, Guo Y, Sheng Q and Shyr Y: Advanced heat map and clustering analysis using heatmap3. Biomed Res Int 2014: 986048, 2014.

16. Dweep H and Gretz N: miRWalk2.0: A comprehensive atlas of microRNA-target interactions. Nat Methods 12: 697, 2015.

17. Shannon P, Markiel A, Ozier O, Baliga NS, Wang JT, Ramage D, Amin N, Schwikowski B and Ideker T: Cytoscape: A software environment for integrated models of biomolecular interaction networks. Genome Res 13: 2498-2504, 2003.

18. Carmona-Saez P, Chagoyen M, Tirado F, Carazo JM and Pascual-Montano A: GENECODIS: A web-based tool for finding significant concurrent annotations in gene lists. Genome Biol 8: R3, 2007.

19. Reiner-Benaim A: FDR control by the BH procedure for two-sided correlated tests with implications to gene expression data analysis. Biom J 49: 107-126, 2007.

20. Zhang Y, Chen Y, Yu J, Liu G and Huang Z: Integrated transcriptome analysis reveals miRNA-mRNA crosstalk in laryngeal squamous cell carcinoma. Genomics 104: 249-256, 2014.

21. Liu C, Li M, Hu Y, Shi N, Yu H, Liu H and Lian H: miR-486-5p attenuates tumor growth and lymphangiogenesis by targeting neuropilin-2 in colorectal carcinoma. Onco Targets Ther 9: 2865-2871, 2016.

22. Ma X, Wei J, Zhang L, Deng D, Liu L, Mei X, He X and Tian J: miR-486-5p inhibits cell growth of papillary thyroid carcinoma by targeting fibrillin-1. Biomed Pharmacother 80: 220-226, 2016.

23. Tian F, Shen Y, Chen Z, Li R, Lu J and Ge Q: Aberrant miR-181b-5p and miR-486-5p expression in serum and tissue of non-small cell lung cancer. Gene 591: 338-343, 2016. 
24. Wang P, Fu T, Wang $X$ and Zhu W: Primary, study of miRNA expression patterns in laryngeal carcinoma by microarray. Lin Chung Er Bi Yan Hou Tou Jing Wai Ke Za Zhi 24: 535-538, 2010 (In Chinese)

25. Ilhan-Mutlu A, Siehs C, Berghoff AS, Ricken G, Widhalm G, Wagner L and Preusser M: Expression profiling of angiogenesis-related genes in brain metastases of lung cancer and melanoma. Tumour Biol 37: 1173-1182, 2016.

26. Sudhakar A and Boosani CS: Inhibition of tumor angiogenesis by tumstatin: Insights into signaling mechanisms and implications in cancer regression. Pharm Res 25: 2731-2739, 2008.

27. Brown CW, Brodsky AS and Freiman RN: Notch3 overexpression promotes anoikis resistance in epithelial ovarian cancer via upregulation of COL4A2. Mol Cancer Res 13: 78-85, 2015.

28. Sablin MP, Dubot C, Klijanienko J, Vacher S, Ouafi L, Chemlali W, Caly M, Sastre-Garau X, Lappartient E, Mariani O, et al: Identification of new candidate therapeutic target genes in head and neck squamous cell carcinomas. Oncotarget 7: 47418-47430, 2016.

29. Parr C, Watkins G, Boulton M, Cai J and Jiang WG: Placenta growth factor is over-expressed and has prognostic value in human breast cancer. Eur J Cancer 41: 2819-2827, 2005.

30. Williams KA, Lee M, Hu Y, Andreas J, Patel SJ, Zhang S, Chines P, Elkahloun A, Chandrasekharappa S, Gutkind JS, et al: A systems genetics approach identifies CXCL14, ITGAX and LPCAT2 as novel aggressive prostate cancer susceptibility genes. PLoS Genet 10: e1004809, 2014.

31. Liu F, Wang X, Li J, Gu K, Lv L, Zhang S, Che D, Cao J, Jin S and Yu Y: miR-34c-3p functions as a tumour suppressor by inhibiting eIF4E expression in non-small cell lung cancer. Cell Prolif 48: 582-592, 2015

32. Li F, Chen H, Huang Y, Zhang Q, Xue J, Liu Z and Zheng F: miR-34c plays a role of tumor suppressor in HEC1-B cells by targeting E2F3 protein. Oncol Rep 33: 3069-3074, 2015.

33. Li YQ, Ren XY, He QM, Xu YF, Tang XR, Sun Y, Zeng MS, Kang TB, Liu N and Ma J: MiR-34c suppresses tumor growth and metastasis in nasopharyngeal carcinoma by targeting MET. Cell Death Dis 6: e1618, 2015.

34. Xu M, Jin H, Xu CX, Bi WZ and Wang Y: MiR-34c inhibits osteosarcoma metastasis and chemoresistance. Med Oncol 31: 972, 2014.

35. Provatopoulou X, Georgiou GP, Kalogera E, Kalles V, Matiatou MA, Papapanagiotou I, Sagkriotis A, Zografos GC and Gounaris A: Serum irisin levels are lower in patients with breast cancer: Association with disease diagnosis and tumor characteristics. BMC Cancer 15: 898, 2015.

36. Yamasaki T, Yoshino H, Enokida H, Hidaka H, Chiyomaru T, Nohata N, Kinoshita T, Fuse M, Seki N and Nakagawa M: Novel molecular targets regulated by tumor suppressors microRNA-1 and microRNA-133a in bladder cancer. Int J Oncol 40: 1821-1830, 2012.

37. Mataki H, Enokida H, Chiyomaru T, Mizuno K, Matsushita R, Goto Y, Nishikawa R, Higashimoto I, Samukawa T, Nakagawa M, et al: Downregulation of the microRNA-1/133a cluster enhances cancer cell migration and invasion in lung-squamous cell carcinoma via regulation of Coronin1C. J Hum Genet 60: 53-61, 2015.
38. Koshizuka K, Hanazawa T, Fukumoto I, Kikkawa N, Matsushita R, Mataki H, Mizuno K, Okamoto Y and Seki N: Dual-receptor (EGFR and c-MET) inhibition by tumor-suppressive miR-1 and miR-206 in head and neck squamous cell carcinoma 62: 113-121, 2017.

39. Zhou W, Fong MY, Min Y, Somlo G, Liu L, Palomares MR, Yu Y, Chow A, O'Connor ST, Chin AR, et al: Cancer-secreted miR-105 destroys vascular endothelial barriers to promote metastasis. Cancer Cell 25: 501-515, 2014.

40. Liu D, Hu X, Zhou H, Shi G and Wu J: Identification of aberrantly expressed miRNAs in gastric cancer. Gastroenterol Res Pract 2014: 473817, 2014.

41. Li M, Liu J, Zhou H, Wu W, Xiao G, Yu Y and Guo L: Expression and clinical significance of CD45RO in laryngeal carcinoma tissue. Lin Chung Er Bi Yan Hou Tou Jing Wai Ke Za Zhi 28: 373-375, 2014 (In Chinese).

42. Saito K, Inagaki K, Kamimoto T, Ito Y, Sugita T, Nakajo S, Hirasawa A, Iwamaru A, Ishikura T, Hanaoka $\mathrm{H}$, et al: MicroRNA-196a is a putative diagnostic biomarker and therapeutic target for laryngeal cancer. PloS One 8: e71480, 2013.

43. Muniyan S, Haridas D, Chugh S, Rachagani S, Lakshmanan I, Gupta S, Seshacharyulu P, Smith LM, Ponnusamy MP and Batra SK: MUC16 contributes to the metastasis of pancreatic ductal adenocarcinoma through focal adhesion mediated signaling mechanism. Genes Cancer 7: 110-124, 2016.

44. Gari HH, DeGala GD, Ray R, Lucia MS and Lambert JR: PRL-3 engages the focal adhesion pathway in triple-negative breast cancer cells to alter actin structure and substrate adhesion properties critical for cell migration and invasion. Cancer Lett 380: 505-512, 2016.

45. Alowayed N, Salker MS, Zeng N, Singh Y and Lang F: LEFTY2 controls migration of human endometrial cancer cells via focal adhesion kinase activity (FAK) and miRNA-200a. Cell Physiol Biochem 39: 815-826, 2016.

46. Lascorz J, Bevier M, V Schönfels W, Kalthoff H, Aselmann H, Beckmann J, Egberts J, Buch S, Becker T, Schreiber S, et al: Association study identifying polymorphisms in CD47 and other extracellular matrix pathway genes as putative prognostic markers for colorectal cancer. Int J Colorectal Dis 28: 173-181, 2013.

47. Zhou L, Chen J, Li Z, Li X, Hu X, Huang Y, Zhao X, Liang C, Wang Y, Sun L, et al: Integrated profiling of microRNAs and mRNAs: microRNAs located on Xq27.3 associate with clear cell renal cell carcinoma. PloS One 5: e15224, 2010.

48. Li Y, Shi X, Yang W, Lu Z, Wang P, Chen Z and He J: Transcriptome profiling of lncRNA and co-expression networks in esophageal squamous cell carcinoma by RNA sequencing. Tumour Biol 37: 13091-13100, 2016. 